\title{
Efectividad del programa educativo "Mi futuro lo decido yo" en la prevención de embarazo no deseado en escolares: Un proyecto de responsabilidad social universitaria
}

Effectiveness of the Educational Program "I decide my future" In the Prevention of Unwanted Pregnancy
in High School Students: A University Social Responsibility Project

Eficácia do programa educacional "Eu decido meu futuro" na prevenção da gravidez indesejada em escolares de ensino médio: Um projeto de responsabilidade social universitária

$\begin{array}{ll}\text { Rinna Pilco Velásquez }^{1 a} & \text { (iD https://orcid.org/0000-0002-4521-1413 } \\ \text { Olga Choque Churata }^{1 a} & \text { (iD https://orcid.org/0000-0002-7604-0514 } \\ \text { Jackeline Flores Flores }^{\text {1b }} & \text { (iD https://orcid.org/ 0000-0002-1740-615X }\end{array}$

Resumen

Objetivo: Determinar la efectividad de un programa educativo para la prevención de embarazo no deseado en estudiantes del nivel secundario de la Institución Educativa Ramón Copaja de la provincia de Tarata en Tacna, durante el año 2019. Material y métodos: Investigación aplicada y prospectiva en 91 estudiantes del nivel secundario. Se aplicó un pre y postest, además de 7 talleres a cargo de estudiantes de quinto año de obstetricia mediante la educación por pares. El análisis de datos fue a través de estadística descriptiva. Resultados: Las edades de los estudiantes se encontraban en un rango de 11 a 18 años. El 50,5\% era de sexo mujer. El nivel de conocimientos sobre salud sexual y reproductiva encontrado en el pretest fue deficiente para el $50 \%$ de los estudiantes de primer, tercer y quinto año; los cuales se elevaron a nivel bueno y excelente en todas las aulas después de la intervención. En lo referente a la dimensión individual: el $83,5 \%$ se sentía seguro de las decisiones que tomaban, el $98,9 \%$ tenía planificado estudiar una carrera profesional, el $79,1 \%$ pensaba en las consecuencias de sus actos, el 5,5\% había consumido alcohol alguna vez, el 4,4 \% había consumido drogas alguna vez, el 20,9 \% tenía enamorado, el 5,5\% había mantenido relaciones sexuales alguna vez, y el 4,4 \% había utilizado algún método anticonceptivo alguna vez. Conclusión: La aplicación del programa educativo incrementó los conocimientos sobre la prevención de embarazo no deseado en los estudiantes del nivel secundario de la Institución Educativa Ramón Copaja y fortaleció las competencias de las estudiantes de la Escuela Profesional de Obstetricia.

Palabras clave: prevención de embarazo no deseado, programa educativo

\begin{abstract}
Objective: To determine the effectiveness of an educational program for the prevention of unwanted pregnancy in secondary school students at Ramón Copaja Public School in the province of Tarata in Tacna-Peru, 2019. Material and methods: This study was an applied and prospective research. A pre and post-test were given to 91 secondary students. Also, 7 workshops by 5 th year Obstetrics students through peer education. The data analysis was through descriptive statistics. Results: Students were aged between 11 to 18 years. $50.5 \%$ were female. The level of knowledge about sexual and reproductive health found in the pretest was deficient for $50 \%$ of the first, third
\end{abstract}

${ }^{1}$ Escuela Profesional de Obstetricia, Universidad Nacional Jorge Basadre Grohmann. Tacna, Perú

a Doctora en Ciencias con mención en Salud Pública

${ }^{\mathrm{b}}$ Magister en Docencia Universitaria y Gestión en Salud Pública 
and fifth year students; which rose to a good and excellent level in all classrooms after the intervention. Regarding the individual dimension: $83.5 \%$ felt confident about the decisions they made, $98.9 \%$ planned to study a professional career, $79.1 \%$ thought about the consequences of their actions, $5.5 \%$ had ever drank alcohol, $4.4 \%$ had ever consumed drugs, $20.9 \%$ had a partner, $5.5 \%$ had ever sex, and $4.4 \%$ had ever used a contraceptive method. Conclusion: The application of the educational program increased the knowledge about preventing unwanted pregnancy in secondary students at Ramón Copaja Public School and strengthened the competences of the students of Professional School of Obstetrics.

Keywords: unwanted pregnancy prevention, educational program

\section{Resumo}

Objetivo: determinar a eficácia de um programa educativo para a prevenção da gravidez indesejada em alunos do ensino médio da escola pública Ramón Copaja da província de Tarata em Tacna, durante o ano de 2019. Material e métodos: Pesquisa aplicada e prospectiva em 91 nível secundário. Foi aplicado um pré e pós-teste, além de 7 oficinas com alunos do quinto ano de Obstetrícia por meio de educação por pares. A análise dos dados deu-se por meio de estatística descritiva. Resultados: As idades dos alunos variaram de 11 a 18 anos. 50,5\% eram mulheres. $O$ nível de conhecimento sobre saúde sexual e reprodutiva encontrado no pré-teste foi deficiente para $50 \%$ dos alunos do primeiro, terceiro e quinto ano; esse nível subiu para bom e excelente em todas as salas de aula após a intervenção. Em relação à dimensão individual: 83,5\% sentia-se confiantes nas decisões que tomavam, 98,9\% planejam estudar a carreira profissional, $79,1 \%$ pensava nas consequências de suas ações, 5,5\% tinha bebido álcool em alguma vez, 4,4\% tinha usado drogas em alguma vez, 20,9\% tinha namorado(a), 5,5\% tinham mantido relações sexuais alguma vez, e 4,4\% tinha usado algum método contraceptivo alguma vez. Conclusão: A aplicação do programa educativo aumentou o conhecimento sobre a prevenção da gravidez indesejada nos alunos do ensino médio da escola pública Ramón Copaja e fortaleceu as competências dos alunos da Escola Profissional de Obstetrícia.

Palavras-chave: prevenção da gravidez indesejada, programa educacional

\section{Introducción}

Los problemas que enfrentan los jóvenes hoy en día en relación con su salud sexual y reproductiva son múltiples y complejos. La UNFPA ${ }^{1}$ refiere que se debe apoyar a la salud sexual y reproductiva de los adolescentes proporcionándoles acceso a una educación sexual integral; así como servicios para prevenir, diagnosticar y tratar las ITS, además de asesoramiento en el tema de planificación familiar.

De acuerdo a la ENDES 20172, 13 de cada 100 adolescentes de entre 15 y 19 años son madres o están embarazadas. Cada día 4 niñas menores de 15 años dan a luz, según la RENIEC. Esta preocupante situación acentúa la importancia de fortalecer los servicios de salud diferenciados para adolescentes.
El departamento de Tacna cuenta con provincias que se encuentran geográficamente situadas en la zona sierra de nuestro país y que no son ajenas a las estadísticas nacionales. De acuerdo a la información del INEI-ENDES 2017, ${ }^{2}$ un dato que alerta es el incremento observado de embarazos en adolescentes de 15 a 19 años de edad, al pasar de 12,7 \% en el año 2016 a 13,4 \% en el año 2017. Este incremento se observa en las zonas urbanas, al pasar de $9,8 \%$ a $10,7 \%$ entre el 2016 y 2017. En las zonas rurales, se pasó de 22,7 $\%$ a $23,2 \%$ entre el 2016 y 2017.

La I.E. Ramón Copaja de Tarata es el colegio que cuenta con el mayor número de adolescentes matriculados de la localidad (91). Se sabe que no existía precedente de alguna intervención efectuada de estas características en los estudiantes, el director de la escuela nos informó 
que las intervenciones realizadas en salud sexual y reproductiva en los adolescentes usualmente constaban de charlas programadas por el establecimiento de salud de la jurisdicción. Otro alcance importante fue que en la escuela a su cargo se presentaron 3 casos de embarazo en adolescentes; razón por la cual dos alumnas dejaron de estudiar y solo una continuó sus estudios. Por tanto, se presenta la urgente necesidad de conocer ¿Cuál es el nivel de conocimientos en salud sexual y reproductiva de los estudiantes del nivel secundario de la Institución Educativa Ramón Copaja de la localidad de Tarata?

Siendo el embarazo adolescente un problema de salud pública ${ }^{3}$, se elaboró un proyecto de responsabilidad social intercultural con el objetivo de determinar la efectividad del programa educativo "Mi futuro lo decido yo" para la prevención de embarazo no deseados en estudiantes del nivel secundario de la Institución Educativa Ramón Copaja de la localidad de Tarata en el año 2019.

\section{Material y métodos}

El presente estudio fue de tipo aplicativo, ya que se desarrolló un proyecto de intervención dirigido a los estudiantes de primero a quinto de secundaria de la I.E. Ramón Copaja de la provincia de Tarata del Departamento de Tacna-Perú en el año 2019. La muestra fue a conveniencia, siendo objeto de estudio todos los estudiantes matriculados para el año 2019. Como criterios de inclusión y exclusión, se consideró solo a aquellos estudiantes (91) que asistieron a las capacitaciones los días programados. El proyecto de intervención fue ejecutado mediante la aplicación de 7 talleres que utilizaron material educativo acorde a la edad de los participantes. Los talleres fueron ejecutados en 3 intervenciones por estudiantes de quinto año de la ESOB. El impacto fue evaluado mediante la aplicación de un pre y postest de acuerdo a los temas abordados. La estrategia educativa utilizada fue la educación entre pares.

En los talleres se abordaron los siguientes temas: 1) autoestima y derechos en salud sexual y reproductiva, 2) toma de decisiones y proyecto de vida, 3) comunicación asertiva: manejo de conflictos, 4) presión de pares, 5) beneficios del inicio tardío de actividades sexuales, 6 ) infecciones de transmisión sexual y 7) métodos anticonceptivos modernos.

La metodología fue expositiva participativa con el uso de videos, material didáctico y el trabajo en equipo. Para la recolección de datos se elaboró una encuesta con el fin de conocer las características sociodemográficas, y datos para medir el nivel de conocimientos pre y posintervención; así como las dimensiones individual, familiar y social respecto a la salud sexual y reproductiva, ${ }^{4}$ las cuales permitieron brindar recomendaciones a los docentes para dar el soporte a los estudiantes.

Para el procesamiento y análisis de datos, se diseñó una hoja de cálculos en Excel 2013, luego se procesaron los datos en SPSS 21. Esto permitió la elaboración de tablas simples, de doble entrada y gráficos. Se utilizó la estadística descriptiva como: frecuencia y porcentaje.

\section{Resultados}

En la Tabla 1 las edades entre las que fluctúan los estudiantes del nivel secundario se encuentran entre los 11 y 18 años; siendo del sexo mujer el $50,5 \%$ y $49,5 \%$ hombres. En la Tabla 2 observamos que, con respecto al nivel de conocimientos sobre temas de salud sexual y reproductiva, se encontró en el pretest que casi la mitad de los estudiantes tenían niveles de conocimientos deficientes. En el postest se mejoró sustancialmente el conocimiento encontrándose incremento en el nivel bueno y excelente en todas las aulas.

En la Tabla 3, se observan datos referentes a tres dimensiones: 1) Dimensión individual: el 83,5 $\%$ se sentía seguro de las decisiones que toma; el $98,9 \%$ tenía planificado estudiar una carrera profesional; el $79,1 \%$ pensaba en las consecuencias de sus actos; el 82,4\% actuaba teniendo en cuenta a sus padres, el 5,5\% había consumido alcohol alguna vez y el 4,4\% había consumido drogas alguna vez, un $37,4 \%$ se refugiaba en las amistades y parejas ante la presencia de problemas; el 20,9 \% tenía enamorado, el 5,5\% había tenido alguna vez 
relaciones sexuales y el 4,4\% había utilizado algún método anticonceptivo alguna vez. 2) Dimensión familiar: el $92 \%$ vivía con sus padres, el 80,2 \% tenía buena comunicación; al $73,6 \%$ sus padres les permitían salir con frecuencia a las fiestas, el 65,9\% les contaba sin temor lo que le pasaba a sus padres y el $62,6 \%$ sentía que sus padres los querían 3 ) Dimensión social: el $18,7 \%$ había visto películas o telenovelas de contenido sexual, el 83,5 \% recibía orientación en su colegio sobre relaciones sexuales y embarazo, el 69,2 \% tenía en cuenta la opinión de sus amigos(as) ante cualquier decisión, el 12,1\% manifestaba que sus amigos habían iniciado o mantenían relaciones sexuales, el 3,3 \% señaló que alguien los motivaba o presionaba a tener relaciones sexuales, el 31,9 \% señaló tener amigos que fumaban y/o consumían algún tipo de alcohol y el $16,5 \%$ conocía personas que consumían o habían consumido drogas.

\section{Discusión}

Mediante la intervención en la localidad de Tarata, pudimos evidenciar la necesidad de este tipo de actividades; puesto que mediante levantamiento previo de datos se observó que la Institución Educativa Ramón Copaja no es ajena a los casos de embarazos en adolescentes. Respecto al nivel de conocimientos en salud sexual y reproductiva, encontramos que casi la mitad de los adolescentes en estudio presentaban un deficiente nivel de conocimientos y que, posterior a la intervención, se mejoró sustancialmente el conocimiento en el nivel bueno y excelente en todas las aulas. De forma semejante, Calderón ${ }^{5}$ obtuvo que el $92 \%$ de los estudiantes presentaron un nivel adecuado de conocimiento sobre planificación familiar después de una intervención educativa como parte de su estudio. El autor concluye que existe una influencia en la aplicación de una estrategia educativa de Salud. Similarmente, Rodríguez ${ }^{6}$ realizó un estudio en el que se encontraron deficiencias en la educación sexual, baja percepción del riesgo de un embarazo o de contraer alguna infección de trasmisión sexual, prácticas de cambio frecuente de parejas, tanto en sexo masculino como en el femenino.

En cuanto a la dimensión individual de los estudiantes de la Institución Educativa Ramos Copaja, podemos detallar que si bien es cierto el $82,4 \%$ manifestó que actuaba teniendo en cuenta a sus padres, encontramos que aun así el 5,5\% consumía algún tipo de bebidas alcohólicas; el 4,4 \% había consumido drogas alguna vez y el $37,4 \%$ se refugiaba en las amistades y parejas ante la presencia de problemas. Datos que difieren de los encontrados en el INSM, en el cual la prevalencia de consumo de alcohol en los adolescentes de las áreas rurales se encontraba en $51,3 \%$, una diferencia de 10,9 puntos porcentuales respecto de la urbana. ${ }^{7}$ Evidenciamos también que el 20,9\% tenía enamorado, siendo los varones los que predominan con esta realidad. El 5,5\% había mantenido alguna vez relaciones sexuales (4 varones y 1 mujer) y el 4,4 \% había utilizado algún método anticonceptivo alguna vez. Estas características demandan atención ante la probabilidad de un embarazo no deseado. En cuanto a la dimensión familiar, evidenciamos que el $92 \%$ vivía con sus padres, solo el $62,6 \%$ sentía que sus padres los querían; el 80,2 \% tenía buena comunicación con ellos y aun así solo el 65,9 \% les contaba sin temor lo que les pasa. Con respecto a la dimensión social, el 18,7 \% había visto películas o telenovelas de contenido sexual, el 69,2 \% tenía en cuenta la opinión de sus amigos(as) ante cualquier decisión, el 12,1 \% manifestaba que sus amigos habían iniciado o tienen relaciones sexuales, el 3,3 $\%$ manifestó que alguien los motiva o presiona a tener relaciones sexuales. Frente a esta última situación, se agregan factores de riesgo: el 31,9\% señaló que tenían amigos que fumaban $\mathrm{y} / \mathrm{o}$ consumían algún tipo de alcohol y el 16,5 \% conocía a personas que consumían o habian consumido drogas. Sin embargo, cabe destacar que el 83,5\% señaló recibir orientación en su colegio sobre relaciones sexuales y embarazo, pero al parecer la información era deficiente.

En conclusión, este estudio nos proporciona evidencia consistente que este tipo de intervención con programas educativos participativos organizados y dictados por grupos de pares tiene efectos sumamente positivos, ya que incrementa los conocimientos en los estudiantes. Resaltamos aquí que son ellos los que construyen en base a sus vivencias conocimientos nuevos y de interés personal. Así mismo, se generó un impacto social, hacia la comunidad beneficiaria en dos etapas. La etapa a corto plazo: mediante la aplicación de instrumentos de evaluación y su correspondiente análisis pudimos corroborar que se elevó el nivel de conocimiento de temas relacionados con salud 
sexual y reproductiva mediante los talleres de intervención; además, se reforzó el tema de autoestima y toma de decisiones asertivas. En la etapa de impacto social a largo plazo: Las disminuciones de los indicadores de embarazos en adolescentes de las zonas rurales no podían ser evidenciadas con una sola intervención. Este es un cambio de conducta que merece un proceso educativo periódico y permanente, por lo que consideramos que se ha dado un primer paso y es ya un compromiso asumido por la ESOB mantener la continuidad de esta intervención durante los siguientes años de estudio.

Respecto al impacto generado en la formación profesional de nuestros estudiantes podemos decir que mediante esta intervención nuestros estudiantes aplicaron sus conocimientos de planeamiento estratégico a través de la elaboración, programación y ejecución de los talleres educativos en uso de técnicas y dinámicas grupales. ${ }^{8}$

\section{Agradecimientos}

Queremos expresar un profundo agradecimiento a las docentes, estudiantes y personal administrativo de la Escuela Profesional de Obstetricia de la Facultad de Ciencias de la salud de la Universidad Nacional Jorge Basadre Grohamnn, así como a los docentes y estudiantes de la Institución Educativa Ramón Copaja por todo el esfuerzo realizado en la ejecución del proyecto de investigación.

\section{Referencias}

1. Fondo de Población de las Naciones Unidas. Salud sexual y reproductiva de los adolescentes [Internet]. [citado 28 de mayo de 2020]. Disponible en: /es/resources/saludsexual-y-reproductiva-de-los-adolescentes

2. Instituto Nacional de Estadística e Informática. Encuesta Demográfica y de Salud FamiliarENDES. Perú, 2017. 398 p. [Internet]. [citado 28 de mayo de 2020]. Disponible en:
https://www.inei.gob.pe/media/MenuRecursivo /publicaciones_digitales/Est/Lib1525/index.ht $\mathrm{ml}$

3. Vulanovic L. Salud del Adolescente - Salud Sexual y Reproductiva [Internet]. Pan American Health Organization / World Health Organization. [citado 5 de junio de 2020]. D i s p o n i b l e e n : https://www.paho.org/hq/index.php?option=co m_content\&view $=$ article\&id=3178: salud-dela d o I e s c e n t e - s a I u d - s e x u a I - y reproductiva\&ltemid=2414\&lang $=e s$

4. Chunga N. Efectividad de un programa educativo «Previniendo el embarazo no deseado en adolescentes» [Internet] [Tesis de Especialidad]. Trujillo-Perú: Universidad Nacional de Trujillo; 2018. 99 p. Disponible en: h t t p s : / / b i t. I y / 2 Y 9 a n $8 \mathrm{~N}$ http://dspace.unitru.edu.pe/handle/UNITRU/11 818

5. Copa E.G.C., Roas J.J.E.C., Espejo Y.E.P., Jiménez K.G.G., Urbano L.C.I., Humpiri J.L.L. Influencia de una estrategia educativa de salud en el nivel de conocimientos y uso de métodos anticonceptivos, en estudiantes del tercer, cuarto y quinto año de secundaria de la Institución Educativa Enrique Paillardelle del Dtto. G.A.L junio-diciem, 2016.Rev. Méd. B a s a d rina. $2017 ; 11$ ( 2 ): $20-5$. http://www.unjbg.edu.pe/revistas/pdf/2017121 5-revista-medica-2.pdf

6. Cabrera D.A.R., Ramos, D.G.S., Palú E.C., Cáceres B.P. Estrategia educativa sobre promoción en salud sexual y reproductiva para adolescentes y jóvenes universitarios. Rev. Cubana de Salud Pública. 2013, 39(1) 161-174. http://scielo.sld.cu/scielo.php?script=sci_arttex t\&pid=S0864-34662013000100015

7. Ministerio de Salud. Documento Técnico: Situación de Salud de los Adolescentes y Jóvenes en el Perú. 2017 / -1a ed. Lima: Ministerio de Salud, 2017. [Internet]. [citado 5 de junio de 2020]. Disponible en: http://bvs.minsa.gob.pe/local/MINSA/4143.pdf

8. Centros de Integración juvenil, A.C. Manual de Técnicas y dinámicas grupales. 2013 [citado en

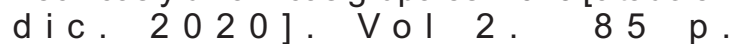
http://www.intranet.cij.gob.mx/Archivos/Pdf/Ma terialDidacticoPreventivo/MANUALDETECNIC ASYDINAMICASGRUPALES.pdf

\section{Correspondencia:}

rpilcov@unjbg.edu.pe
Fecha de recepción: 21 de septiembre del 2020 Fecha de aceptación: 10 de diciembre del 2020 


\section{Tabla 1}

Año de estudios según edad y sexo de los estudiantes

\begin{tabular}{|c|c|c|c|c|c|c|c|c|c|c|c|c|c|c|c|c|c|c|c|c|}
\hline \multirow[t]{4}{*}{ Edad } & \multicolumn{20}{|c|}{ Año de estudio } \\
\hline & \multicolumn{4}{|c|}{ Primero } & \multicolumn{4}{|c|}{ Segundo } & \multicolumn{4}{|c|}{ Tercero } & \multicolumn{4}{|c|}{ Cuarto } & \multicolumn{4}{|c|}{ Quinto } \\
\hline & \multicolumn{2}{|c|}{ Femenino } & \multicolumn{2}{|c|}{ Masculino } & \multicolumn{2}{|c|}{ Femenino } & \multicolumn{2}{|c|}{ Masculino } & \multicolumn{2}{|c|}{ Femenino } & \multicolumn{2}{|c|}{ Masculino } & \multicolumn{2}{|c|}{ Femenino } & \multicolumn{2}{|c|}{ Masculino } & \multicolumn{2}{|c|}{ Femenino } & \multicolumn{2}{|c|}{ Masculino } \\
\hline & $n$ & $\%$ & $n$ & $\%$ & $n$ & $\%$ & $n$ & $\%$ & $n$ & $\%$ & $n$ & $\%$ & $n$ & $\%$ & $n$ & $\%$ & $n$ & $\%$ & $n$ & $\%$ \\
\hline 11 & & & 1 & 1,1 & & & & & & & & & & & & & & & & \\
\hline 12 & 7 & 7,7 & 12 & 13,2 & & & & & & & & & & & & & & & & \\
\hline 13 & 3 & 3,3 & 3 & 3,3 & 5 & 5,5 & 5 & 5,5 & & & & & & & & & & & & \\
\hline 14 & & & & & 3 & 3,3 & 1 & 1,1 & 8 & 8,8 & 8 & 8,8 & 1 & 1,1 & 1 & 1,1 & & & & \\
\hline 15 & & & & & & & & & 2 & 2,2 & & & 9 & 9,9 & 6 & 6,6 & & & 1 & 1,1 \\
\hline 17 & & & & & & & & & & & & & & & & & 2 & 2,2 & & \\
\hline 18 & & & & & & & & & & & & & & & & & & & 1 & 1,1 \\
\hline \multirow[t]{2}{*}{ Total } & 10 & 11 & 16 & 17,6 & 8 & 8,8 & 6 & 6,6 & 11 & 12,1 & 8 & 8,8 & 10 & 11 & 8 & 8,8 & 7 & 7,7 & 7 & 7,7 \\
\hline & \multicolumn{4}{|c|}{26} & \multicolumn{4}{|c|}{14} & \multicolumn{4}{|c|}{19} & \multicolumn{4}{|c|}{18} & \multicolumn{4}{|c|}{14} \\
\hline
\end{tabular}

\section{Tabla 2}

Nivel de conocimientos en temas de salud sexual y preproductiva pretest y postest de los estudiantes

\begin{tabular}{|c|c|c|c|c|c|c|c|c|c|c|c|c|c|c|c|c|c|c|c|c|}
\hline & \multicolumn{4}{|c|}{ Primero } & \multicolumn{4}{|c|}{ Segundo } & \multicolumn{4}{|c|}{ Tercero } & \multicolumn{4}{|c|}{ Nivel de conocimiento } & \multicolumn{4}{|c|}{ Quinto } \\
\hline & \multicolumn{2}{|c|}{ Pre test } & \multicolumn{2}{|c|}{ Post test } & \multicolumn{2}{|c|}{ Pre test } & \multicolumn{2}{|c|}{ Post test } & \multicolumn{2}{|c|}{ Pre test } & \multicolumn{2}{|c|}{ Post test } & \multicolumn{2}{|c|}{ Pre test } & \multicolumn{2}{|c|}{ Post test } & \multicolumn{2}{|c|}{ Pre test } & \multicolumn{2}{|c|}{ Post test } \\
\hline & $n$ & $\%$ & $n$ & $\%$ & $n$ & $\%$ & $n$ & $\%$ & $n$ & $\%$ & $n$ & $\%$ & $n$ & $\%$ & $n$ & $\%$ & $n$ & $\%$ & $n$ & $\%$ \\
\hline Deficiente & 15 & 57,69 & 8 & 30,77 & 4 & 28,57 & 2 & 15,38 & 11 & 57,89 & 0 & 0,00 & 5 & 27,77 & 1 & 5,88 & 6 & 42,85 & 1 & 6,66 \\
\hline Regular & 7 & 26,92 & 9 & 34,62 & 5 & 35,71 & 3 & 23,08 & 3 & 15,79 & 3 & 15,00 & 4 & 22,22 & 5 & 29,41 & 2 & 14,28 & 3 & 20 \\
\hline Bueno & 4 & 15,38 & 5 & 19,23 & 4 & 28,57 & 7 & 46,15 & 4 & 21,05 & 4 & 20,00 & 8 & 44,44 & 5 & 29,41 & 4 & 28,57 & 5 & 33,33 \\
\hline Excelente & & 0 & 4 & 15,38 & 1 & 7,14 & 2 & 15,38 & 1 & 5,26 & 12 & 65,00 & 1 & 5,55 & 7 & 35,24 & 2 & 14,28 & 5 & 40 \\
\hline Total & 26 & & 26 & & 14 & & 14 & & 19 & & 19 & & 18 & & 18 & & 14 & & 14 & \\
\hline
\end{tabular}

Deficiente: 0 - 10, Regular: 11 - 13, Bueno: 14 - 16, Excelente: 17 - 20 (3)

\section{Figura 1}

Nivel de conocimiento sobre temas de salud sexual y reproductiva de los estudiantes de la I. E. Ramón Copaja de Tarata

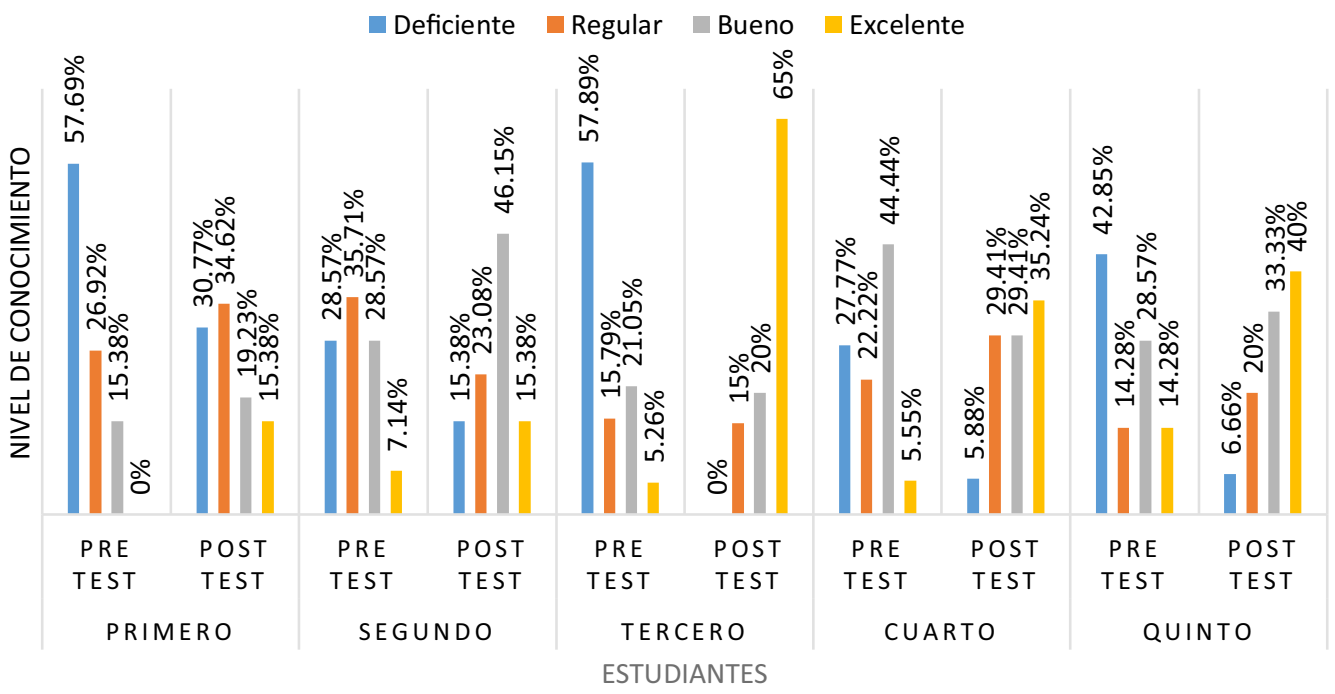

Revista Médica Basadrina, 2020; 14(2):3-9. 
Efectividad del programa educativo "Mi futuro lo decido yo" en la prevención de embarazo no deseado en escolares: Un proyecto de responsabilidad social universitaria

\section{Tabla 3}

Características de la dimensión individual, familiar y social según sexo de los estudiantes

\begin{tabular}{|c|c|c|c|c|c|c|c|c|}
\hline \multirow{3}{*}{ Dimensiones } & \multicolumn{4}{|c|}{ Femenino } & \multicolumn{4}{|c|}{ Masculino } \\
\hline & \multicolumn{2}{|c|}{ Si } & \multicolumn{2}{|c|}{ No } & \multicolumn{2}{|c|}{$\mathrm{Si}$} & \multicolumn{2}{|c|}{ No } \\
\hline & $\mathbf{N}^{\circ}$ & $\%$ & $\mathbf{N}^{\circ}$ & $\%$ & $\mathrm{~N}^{\circ}$ & $\%$ & $\mathrm{~N}^{\circ}$ & $\%$ \\
\hline DIMENSIóN INDIVIDUAL & & & & & & & & \\
\hline ¿Me siento segura de las decisiones que tomo? & 37 & 40,7 & 09 & 9,9 & 39 & 42,9 & 6 & 6,6 \\
\hline $\begin{array}{l}\text { ¿Cuando termine mis estudios secundarios, me gustaría } \\
\text { estudiar una carrera profesional? }\end{array}$ & 45 & 49,5 & 01 & 1,1 & 45 & 49,5 & 0 & 0 \\
\hline ¿Siempre pienso en las consecuencias de mis actos? & 39 & 42,9 & 07 & 7,7 & 33 & 36,3 & 12 & 13,2 \\
\hline ¿Actuó teniendo en cuenta a mis padres? & 34 & 37,4 & 12 & 13,2 & 41 & 45,1 & 4 & 4,4 \\
\hline ¿Consumiste o consumes algún tipo de bebidas alcoholes? & 2 & 2,2 & 44 & 48,4 & 3 & 3,3 & 42 & 46,2 \\
\hline ¿Consumiste drogas alguna vez por curiosidad? & 2 & 2,2 & 44 & 48,4 & 2 & 2,2 & 43 & 47,3 \\
\hline $\begin{array}{l}\text { ¿Cuándo tengo problemas me refugio con mis amigos y/o } \\
\text { novio }\end{array}$ & 19 & 20,9 & 27 & 29,7 & 15 & 16,5 & 30 & 33,0 \\
\hline ¿Tengo enamorado y/o novio? & 8 & 8,8 & 38 & 41,8 & 11 & 12,1 & 34 & 37,4 \\
\hline ¿Has tenido alguna vez relaciones sexuales? & 1 & 1,1 & 45 & 49,5 & 4 & 4,4 & 41 & 45,1 \\
\hline ¿Has utilizado algún método anticonceptivo alguna vez? & 1 & 1,1 & 45 & 49,5 & 3 & 3,3 & 42 & 46,2 \\
\hline $\begin{array}{l}\text { DIMENSIóN FAMILIAR } \\
\text { ¿Vives con tus padres? }\end{array}$ & 42 & 46,2 & 4 & 4,4 & 42 & 46,2 & 3 & 3,3 \\
\hline ¿Existe conflictos con tu familia? & 10 & 11,0 & 36 & 39,6 & 9 & 9,9 & 36 & 39,6 \\
\hline ¿Tienes una buena comunicación con tus padres? & 36 & 39,6 & 10 & 11,0 & 37 & 40,7 & 8 & 8,8 \\
\hline $\begin{array}{l}\text { ¿Tus padres le permiten salir con frecuencia a fiestas } \\
\text { bailes? }\end{array}$ & 8 & 8,8 & 38 & 41,8 & 16 & 17,6 & 29 & 31,9 \\
\hline ¿Le cuentas sin miedo lo que te pasa a tus padres? & 26 & 28,6 & 20 & 22,0 & 34 & 37,4 & 11 & 12,1 \\
\hline $\begin{array}{l}\text { ¿Mis padres me hacen sentir que me quieren a través de } \\
\text { besos, caricias o palabras? }\end{array}$ & 26 & 28,6 & 20 & 22,0 & 31 & 34,1 & 14 & 15,4 \\
\hline $\begin{array}{l}\text { DIMENSIóN SOCIAL } \\
\text { ¿Has visto películas o telenovelas de contenido sexual? }\end{array}$ & 4 & 4.4 & 42 & 46.2 & 13 & 14.3 & 32 & 35.2 \\
\hline $\begin{array}{l}\text { ¿Recibes orientación en tu colegio sobre relaciones } \\
\text { sexuales y embarazo? }\end{array}$ & 41 & 45.1 & 5 & 5.5 & 35 & 38.5 & 10 & 11.0 \\
\hline $\begin{array}{l}\text { ¿Tienes en cuenta la opinión de tus amigos(as) ante } \\
\text { cualquier decisión? }\end{array}$ & 35 & 38.5 & 11 & 12.1 & 28 & 30,8 & 17 & 18,7 \\
\hline ¿Tus amigos han iniciado o tienen relaciones sexuales? & 6 & 6,6 & 40 & 44,0 & 5 & 5,5 & 40 & 44,0 \\
\hline ¿Alguien te motiva o presiona a tener relaciones sexuales? & 1 & 1,1 & 45 & 49,5 & 2 & 2,2 & 43 & 47,3 \\
\hline ¿Participas en algunas actividades en algún grupo religioso? & 27 & 29,7 & 19 & 20,9 & 19 & 20,9 & 26 & 28,6 \\
\hline $\begin{array}{l}\text { ¿'Tienes amigos que fuman y/o consumen algún tipo de } \\
\text { alcohol? }\end{array}$ & 13 & 14,3 & 33 & 36,3 & 16 & 17,6 & 29 & 31,9 \\
\hline $\begin{array}{l}\text { ¿Conoces a personas que consumen o han consumido } \\
\text { drogas? }\end{array}$ & 8, & 8,8 & 38 & 41,8 & 7 & 7,7 & 38 & 41,8 \\
\hline
\end{tabular}

